\title{
Impact of red blood cell storage under the blood bank conditions on their reactivity with autoantibodies
}

\author{
KATARZYNA GMEREK ${ }^{1,2}$, JADWIGA FABIJAŃSKA-MITEK ${ }^{1}$, ADRIANNA ŁONIEWSKA-LWOWSKA ${ }^{l}$
}

${ }^{1}$ Department of Immunohaematology, The Medical Centre of Postgraduate Education, Warsaw, Poland

${ }^{2}$ Regional Centre of Blood Transfusion, Warsaw, Poland

\begin{abstract}
Background: Due to the contemporary red blood cell $(R B C)$ storage methods e.g. appropriate preservative solutions and adequate temperature, the storage duration for so-called erythrocyte concentrate (EC) has been extended to 42 days. Depending on the method of EC preparation, they are non- or leucoreduced. The clinical retrospective studies appearing over the past decade have pointed out the potential risk of adverse transfusion outcomes, especially in the case of long stored and non-leucoreduced RBCs. The increased haemolysis as well as the appearing immunomodulatory processes can depend on the antigen-antibody reaction. After 120 days in human bloodstream, senescent RBCs are removed by the spleen macrophages in autoimmunological process recalling the warm IgG-RBC interaction, characteristic for the autoimmune haemolytic anaemia (AIHA).

Aim of this study was to evaluate the mode of interaction of fresh vs stored RBCs with autoantibodies of AIHA suffering patients.

Material and methods: Autoantibodies from 10 blood samples of AIHA patients were eluted and combined. The autoantibody titre was assessed against 24 fresh, leucoreduced RBC samples and 24 stored for six weeks, non-and leucoreduced RBCs. Results of titration study by the indirect antiglobulin test (IAT) were presented as score values. Morphological parameters of 60 blood samples from leukoreduced ECs were estimated at the beginning and at the end of RBC storage period. For statistical analysis, the t-student test was applied for groups of related (morphology) as well as for groups of unrelated (autoantibody reactivity). Results: Autoantibody reactivity against fresh RBCs was statistically lower than against 6 weeks stored leucoreduced or not RBCs $(p<0.001)$. MCV of fresh RBCs was significantly lower than of those stored $(p<0.001)$.

Conclusion: Six weeks stored RBCs, irrespectively on the leucocyte presence in the ECs, reveal stronger activity with autoantibodies when compared with fresh RBCs. This observation can be explained by the direct changes in the antigenic structure or their increased accessibility related with the enlarging $R B C$ volume during the storage period.
\end{abstract}

Key words: $R B C$ senescence, autoantibodies against $R B C s, R B C$ storage conditions.

(Centr Eur J Immunol 2012; 37 (3): 243-246)

\section{Introduction}

After removing plasma from the unit of donated blood, RBCs intended for transfusion are kept in PCV bags, at $4-6^{\circ} \mathrm{C}$ as so-called erythrocyte concentrates (ECs). Application of suitable preservation solutions, for example CPD (citrate-phosphate-dextrose-adenine) + SAGM (saline-ade- nine-glucose-mannitol) allows RBCs to be stored up to 42 days $[1,2]$. EC can be stored together with leucocytes or can also be deprived of those cells by filtration. Retrospective clinical studies point out the adverse transfusion effects of long stored non- or leucoreduced RBCs on the recipient's recovery [3-9]. These concerns mostly patients

Correspondence: Ass. Prof. Jadwiga Fabijańska-Mitek, PhD, Department of Immunohaematology, The Medical Centre of Postgraduate Education, Marymoncka 99/103, 01-813 Warsaw, tel. +48 2256938 20, fax +48 2256938 29, e-mail: biofizyka@cmkp.edu.pl 
of the cardiology departments, with acute coronary syndrome, ischemic heart disease or after coronary interventions, who are particularly susceptible for effects of hypoxia, electrolyte imbalance, activation of thrombosis, presence of proinflammatory factors and haemolysis. The correlation was observed between long stored non-leucoreduced RBCs transfusions and an increased intensive care unit length of stay, sepsis, acute renal dysfunction, transfusionrelated acute lung injury, multiorgan failure and also increased in-hospital and out-of-hospital mortality. In recent years there has been increasing interest in the idea that allogeneic blood transfusions may be associated with so-called transfusion-related immunomodulation (TRIM) that refers to increase in the risk of infections, cancer recurrence and autoimmunisation.

It is well known that during the storage under blood bank conditions, RBCs undergo a number of biochemical, morphological and metabolic changes known as "storage lesions" [10-15]. For example, it is well described, that due to accumulation of lactic acid in the blood bag, the $\mathrm{pH}$ decreases, and this increases phosphatase enzyme activity, which results in 2,3-diphosphoglycerol (2,3-DPG) degradation. Low level of 2,3-DPG results in increased haemoglobin affinity for oxygen and thereby causes diminished delivery of oxygen to tissues. During RBCs storage period there are also changes in ATP level what reflects on reology of erythrocyte, loss of S-nitrosothiol-haemoglobin (SNO-Hb) and thereby decreased NO production and further vasodilatation, as well as membranous band 3 protein and some other proteins rearrangements, that may lead to erythrocyte disintegration and haemolysis.

According to some scientists, in vivo RBC lifespan is regulated by autoimmunological process [16-20]. Under the erythrocyte aging, the amount of some surface molecules e.g. sialic acid is reduced, while other RBC membrane structures like band 3 and $\mathrm{Rh}$ proteins emerge. Therefore, it is postulated that erythrocytes obtain new antigenic properties and in reaction with natural autoantibodies are removed from bloodstream by spleen macrophages after around 120 days.

Similarly act warm IgG autoantibodies in AIHA, however the RBC lifespan is significantly shortened and sometimes warm IgG autoantibodies preferably interact with the old rather than young patient's RBCs.

In this study we aimed to evaluate changes of interaction of fresh vs. stored donor RBCs with autoantibodies of AIHA suffering patients.

\section{Material and methods}

\section{Red blood cells sampling}

RBC samples were obtained from the drains of the EC bags containing CPD + SAGM. For the reaction with autoantibodies analysis, RBC samples obtained from 108 ECs were used: 3 groups of 24 samples of fresh ( 3 to 5 days after donation) leucoreduced ECs and non- and leucoreduced 6 weeks stored ECs, then 3 groups of 12 samples of fresh leukoreduced ECs and non- and leukoreduced 3 weeks stored ECs.

In order to perform the morphological analysis, 60 leucoreduced RBC samples were taken at the beginning and at the end of their storage and measurement was carried out using the haematological analyzer (Beckman Coulter, USA).

\section{Autoantibodies}

The serological diagnosis of warm-type AIHA in 10 patients was based on a positive gel direct antiglobulin test with monospecific anti-human globulin serum anti-IgG (DiaMed-ID, Switzerland). The RBC eluates were prepared by the acid elution procedure. One volume of washed packed RBCs was mixed with two volumes of an acid/EDTA buffer ( $\mathrm{pH} 1.5$ ), incubated for 2 minutes, then centrifuged and the supernatant (eluate) neutralized with the TRIS buffer. Studied RBC autoantibodies were typical, non-specific, what means that they were panreactive and gave the same reactions with all panel RBCs routinely used in serological laboratories, regardless of donors' phenotypes. The eluates from each sample were combined and used for titration study.

\section{Autoantibody reactivity}

Two milliliters of mixture of eluates were prepared in serial dilutions from $1 / 2$ to $1 / 256$ with PBS and studied by the microcolumn gel-indirect antiglobulin test with all 108 samples of RBCs according to the manufacturer's instruction (IAT; LISSCoombs, DiaMed-ID, Switzerland). Obtained results were presented as the score values for the last 3 dilutions, where the reactions were observed. IAT results are semiquantitative and strength of reactions usually are noted as $4+, 3+, 2+, 1+, 1+_{\text {weak }}$, negative or as respective numbers $12,10,8,5,3,0$. The way of examination can be modified. We introduced additional grades $2.5+$, $1.5+,+/-$ and appropriate numbers $(9,7,2)$. In our titration study made parallel with RBCs from three EC populations, first dilutions of autoantibodies ex. 1/2, 1/4, 1/8 gave equal results $4+$, then differentiated in three next dilutions ex. $1 / 16,1 / 32,1 / 64$ and these were counted as score. Reactions of agglutination were fixed and easy to read because we used microcolumn gel IAT.

\section{Statistics}

For statistical analysis, the t-student test was applied for groups of related (morphology) as well as for groups of unrelated (autoantibody reactivity with RBCs).

\section{Results}

The determined autoantibody titer for fresh RBCs was 32 or 64 , and in case of 6 weeks stored RBCs was higher and was 64 or 128 . The score values were: $10.25 \pm 3.45$ for 


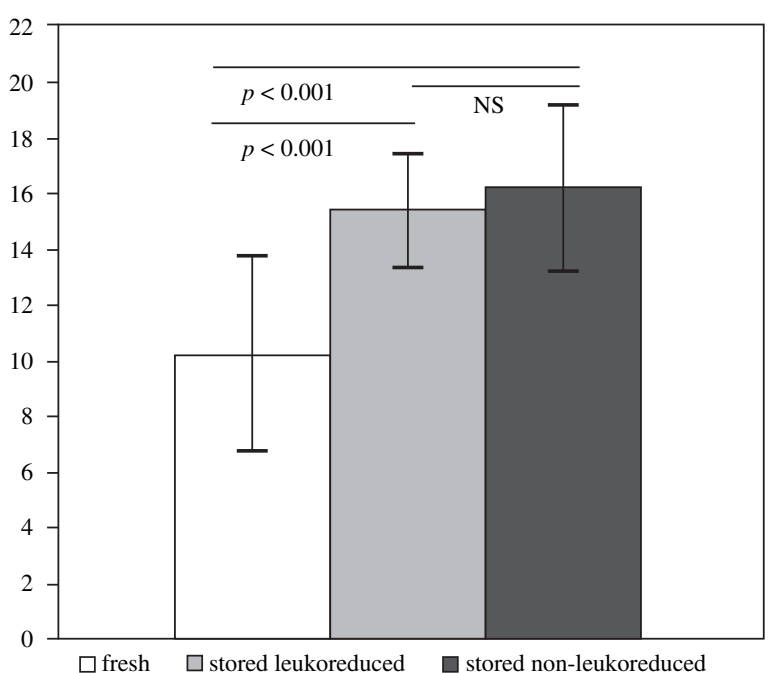

\begin{tabular}{|l|r|r|r|}
\hline Score & 10.25 & 15.41 & 16.25 \\
\hline SD & 3.45 & 2.08 & 3.01 \\
\hline
\end{tabular}

Fig. 1. Reactivity of autoantibodies with red blood cells from three EC populations, each of 24 samples: fresh, six weeks stored leukoreduced and non-leukoreduced $(p<0.001$ for difference between fresh and stored RBCs)

fresh RBCs, $15.41 \pm 2.08$ for stored non-leucoreduced and $16.25 \pm 3.01$ for stored leucoreduced RBCs. This observed difference in RBC-autoantibody reactivity of fresh vs. stored RBCs was statistically significant, with $p<0.001$. There was a slight increase in autoantibody titer and mean score value of stored, non-leucoreduced RBCs in comparison with stored leucoreduced RBCs, however, this difference was statistically not significant (Fig. 1). Comparing autoantibody reactivity of fresh vs. 3 weeks stored RBCs we noticed very slight increase against non-leukoreduced RBCs but it was statistically not significant and in these three groups score values were almost equal.

Analysis of morphological parameters revealed statistically significant difference in MCV of short (3-5 days) vs. long (6 weeks) stored RBCs: $91.5 \pm 4.08$ fL vs. $95.4 \pm 4.06$ $(p<0.001)$ (Fig. 2).

\section{Discussion}

Preparation process and storage of all of the components of whole blood, including RBCs depends on the local legislation and process is strictly controlled. The last verification criterion, according FDA requirements is determining the validity of the storage period basing on method of the survival of labeled stored RBCs in the volunteers bloodstream. At the last day of the EC unit storage, RBCs should be safe and effective like on the day of donation. It is established that lowered 2,3-DPG, ATP and RBC reological properties are recovered within recipient's blood circulation in several hours after transfusion. Results of our study showed

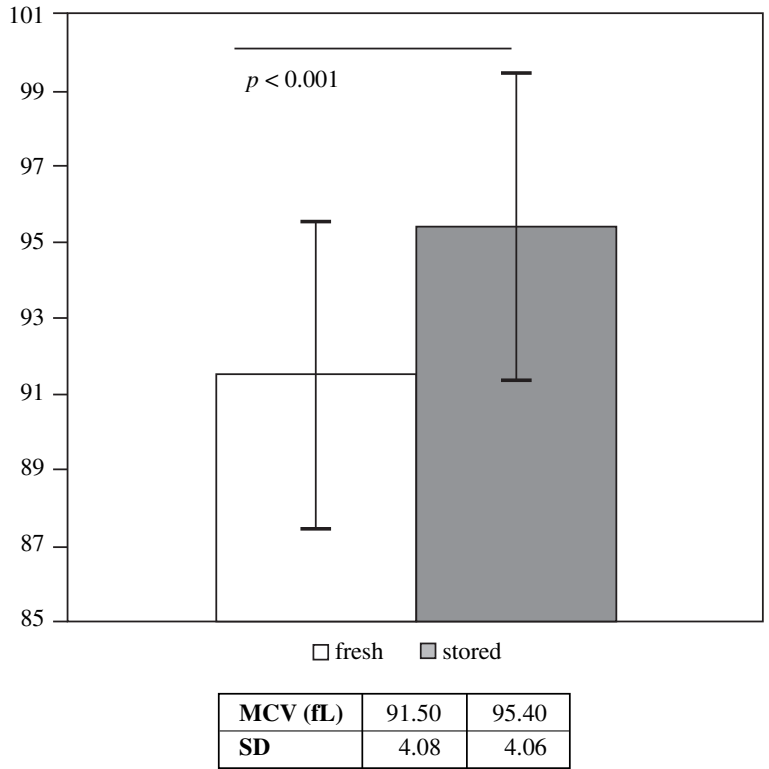

Fig. 2. Medium corpuscular volume (MCV) of red blood cells from 60 units of erythrocyte concentrates measured at the beginning and at the end of storage period $(p<0.001)$

that during storage period, reactivity of RBCs with autoantibodies was increased. It could indicate the direct changes in the antigenic structure. However, the alteration of RBCautoantibody reactivity was not visibly dependent on the presence of leucocytes, their products e.g. proteolityc enzymes, that could be responsible for the RBC membrane modification. On the other hand, there was a significant increase in $\mathrm{MCV}$ values in $6^{\text {th }}$ week of $\mathrm{RBC}$ storage. Increased MCV of RBCs can cause easier accessibility of the antigen determinants for autoantibodies. Regardless of the reason of observed stored RBC changes, their increased reactivity with pathological or physiological autoantibodies can lead to the intensified removal of transfused erythrocytes from recipients bloodstream and in consequence adverse outcomes of the blood transfusion.

This study was supported by 502-1-26-01-11 CMKP grant and was performed with official consent of the Research Bioethics Committee.

\section{References}

1. Murphy M, Wallis J (2009): Red cell transfusion. In: ABC of Transfusion. Fourth Edition. Contreras M (ed.). Wiley-Blackwell, Oxford, 15-21.

2. Łętowska M (2011): Medyczne zasady pobierania krwi, oddzielania składników i wydawania, obowiązujące w jednostkach organizacyjnych publicznej służby krwi. Instytut Hematologii i Transfuzjologii, Warszawa.

3. Corwin HL (1999): First, do not harm! Chest. Blood Transfusion 116: 1149-1150. 
4. Ho J, Sibbald WJ, Chin-Yee IH (2003): Effects of storage on efficacy of red cell transfusion: when is it not safe? (scientific reviews). Crit Care Med 31: S686-S697.

5. Al-Sarraf A, Fowler RA (2005): Is blood transfusion harmful in patients with acute coronary syndromes? JAMC 172: 182.

6. Kiraly LN, Underwood S, Differding JA, et al. (2009): Transfusion of aged packed red blood cells results in decreased tissue oxygenation in critically injured trauma patients. J Trauma 67: 29-32.

7. Weinberg JA, McGwin Jr G, Griffin LG, et al. (2008): Age of transfused blood: an independent predictor of mortality despite universal leukoreduction. J Trauma 65: 279-284.

8. Zimrin AB, Hess JR (2009): Current issues relating to the transfusion of stored red blood cells. Vox Sang 96: 93-103.

9. Zubair A C (2010): Clinical impact of blood storage lesions. Am J Hematol 852: 117-122

10. Hamasaki N, Yamamoto M (2000): Red blood cell function and blood storage. Vox Sang 79: 191-197.

11. De Rosa MC, Carelli Alinovi C, Galtieri A, et al. (2007): The plasma membrane of erythrocytes plays a fundamental role in the transport of oxygen, carbon dioxide and nitric oxide and in the maintenance of the reduced state of the heme iron. Gene 398: 162-171.

12. Endeward V, Cartron J-P, Ripoche P, et al. (2008): RhAG protein of the rhesus complex is a $\mathrm{CO} 2$ channel in the human red cell membrane. FASEB J 22: 64-73.

13. Isbell TS, Sun Ch-W, Wu L-Ch, et al. (2008): SNO-hemoglobin is not essential for red blood cell-dependent hypoxic vasodilation. Nat Med 14: 773-777.

14. van de Watering L (2011): Red cell storage and prognosis. Vox Sang 100: 36-45.

15. Relevy H, Koshkaryev A, Manny N, et al. (2008): Blood banking-induced alteration of red blood cell flow properties. Transfusion 48: 136-146

16. Garratty G: (1996): Novel mechanisms for immune destriction of circulating autologous cells. In: Autoimmune disorders of blood. Ed. Silberstein LE. AABB, Bethesda: 79-114.

17. Garratty G: (1991):Target antigens for red-cell-bound autoantibodies. In: Clinical and basic science aspects of immunohematology. Ed. Nance SJ. AABB, Arlington: 33-72.

18. Kay MM (1992): Senescent cell antigen and band 3 in aging and disease. In: Progress in Cell Research. Ed. Bamberg E, Passow H. Elsevier Science Publishers, 245-250.

19. Kay MM, Wyant T, Goodman J (1994): Antibodies to band 3 during aging and disease and aging interventions. Ann NY Acad Sci 719: 419-447.

20. Leddy JP, Wilkinson SL, Kissel GE, et al. (1994): Erythrocyte membrane protein reactive with $\mathrm{IgG}$ (warm-reacting) anti-red blood cell autoantibodies: II. Antibodies coprecipitating band 3 and glycophorin A. Blood 84: 650-656. 